\title{
Theoretical optimization of the self-organized growth of nanoscale arrays through a figure of merit
}

\author{
E. Vasco ${ }^{a)}$ \\ Institut für Festkörperforschung, Forschungszentrum Jülich, D-52425 Jülich, Germany
}

(Received 24 February 2004; accepted 18 August 2004)

\begin{abstract}
A figure of merit is proposed in order to optimize the self-organized growth of nanoscale elements into one-/two-dimensional arrays via a fine selection of the deposition/annealing conditions. This figure of merit has been designed to account for the most significant defects inherent in such arrays. Its versatility has been studied by kinetic Monte Carlo simulations of self-organized growth of metal clusters on the $7 \times 7$ reconstructed $\mathrm{Si}(111)$ surface. The optimization relationships between deposition/annealing conditions and characteristics of the metal-on- $\operatorname{Si}(111) 7 \times 7$ systems are obtained and analyzed within the framework of competition between thermodynamical tendencies and kinetic limitations. (C) 2004 American Institute of Physics. [DOI: 10.1063/1.1807032]
\end{abstract}

In recent years the subject of self-organization in inorganic materials has emerged as a frontier topic in the field of materials science. Taking advantage of the self-assembling growth on surfaces with a periodic distribution of adsorption sites (such as highly-corrugated, ${ }^{1,2}$ stress-modulated, ${ }^{3}$ or vicinal surfaces, ${ }^{4}$ or those with a dislocation network ${ }^{5}$ ), oneor two-dimensional (1D/2D) arrays of nanometer-sized elements can be fabricated. Beyond the low coverage regimes, the lifetime and versatility of such arrays should be prolonged by inhibiting the early coalescence of arrayed elements via self-patterning mechanisms, ${ }^{6}$ which constitute "a second line of defense." These latter mechanisms, which are very different from artificial nanopatterning techniques such as lithography or mechanical milling, make use of instabilities and kinetic limitations operating in these systems to confine the nucleation areas and thus to retard the coalescence regimes.

Once the manufacturing procedures by self-organization/ self-patterning have been established, it is necessary to optimize the deposition conditions to improve the ensemble features (quality) of such arrays by minimizing their defects. From the assumption of a grid template, which describes the array symmetry, three major array defects can be considered: (i) misalignment defect (with density $\alpha$ ) that takes place when some elements nucleate out of the expected sites; (ii) filling defect $(\beta)$ occurring when some arrayed elements are missing; and (iii) a uniformity defect connected to a broad element size distribution. The two first defects are analogous to Frenkel and Schottky point defects in crystalline lattices, respectively, and can be evaluated according to their corresponding defect densities ${ }^{7}(\alpha \leqslant 1$ and $\beta \leqslant 1)$. On the other hand, the third defect is described through the quadratic deviation of the size $(n)$ distribution: $\omega=\left\langle[n-\langle n\rangle]^{2}\right\rangle^{1 / 2}$, where \langle\rangle denotes the size averaged over the arrayed element population. Because the defects are not independent of each other in practice, a figure of merit $(Q)$ can be defined, on the basis of simultaneous minimization of the defect densities and with the goal of improving the array quality, as

\footnotetext{
${ }^{a}$ Current address: Swiss Federal Institute of Technology, Lausanne (EPFL), Ceramic Laboratory, IMX-D Ecublens, CH-1015 Lausanne, Switzerland; electronic mail: enrique.vasco@epfl.ch
}

$$
Q \propto g(1 / \omega)(1-\alpha)(1-\beta),
$$

where $g(1 / \omega)$ is a generic function that adjusts the statistical weight of the uniformity defect for highly regular arrays (with $\omega \ll 1$ ).

In this letter, $Q$ is used, for example, to optimize the deposition conditions of metal cluster arrays on $7 \times 7$ reconstructed $\mathrm{Si}(111)$ surfaces, a research topic of increasing scientific interest. ${ }^{1,2,8}$ This work is intended to complement previous experimental studies. ${ }^{1,2}$

The $\operatorname{Si}(111) 7 \times 7$ surface is described as a honeycomb structure formed by two large ( $\gamma=24.5$ atoms) triangular half cells with alternate stacking faults (fault, $f$, and unfault, $u$, half cells), which are separated by high energy barriers $(\sim 0.7-1.0 \mathrm{eV})^{9}$ at the dimer row and corner hole sites. The organizational capacity on this surface resides in an adsorption probability differentiated by half cell types (with a higher preference for the faulted half) and in the confinement role played by the barriers at the dimer rows. Such barriers limit the inter-half cell diffusion, preventing cluster coalescence at moderated temperatures. Thus, the balance between kinetic limitations at finite relaxation times and thermodynamical tendencies rules the spontaneous arrangement of metal adsorbates on $\mathrm{Si}(111) 7 \times 7$. This balance can be tuned by varying the deposition/annealing conditions (i.e., temperature, $T$, deposition rate, $F$, and coverage, $\theta$ ) as a function of the system intrinsic characteristics (see below).

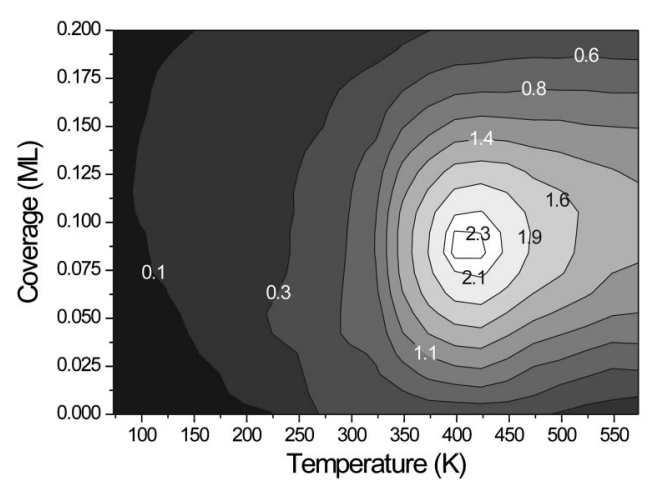

FIG. 1. $Q(T, \theta)$ surface (represented as level curves) calculated by kMC simulations, assuming a prototype set of parameters $\left(E_{s}^{u}=0.75 \mathrm{eV}, \Delta E\right.$ $=0.10 \mathrm{eV}, E_{b}=0.10 \mathrm{eV}, F=0.01 \mathrm{ML} / \mathrm{s}, n_{\max }=6$, and $i^{*}=1$ ) within the range of characteristic values of diffusion and aggregation of metal adsorbates on the $\mathrm{Si}(111) 7 \times 7$ surface (Refs. 11 and 12). 

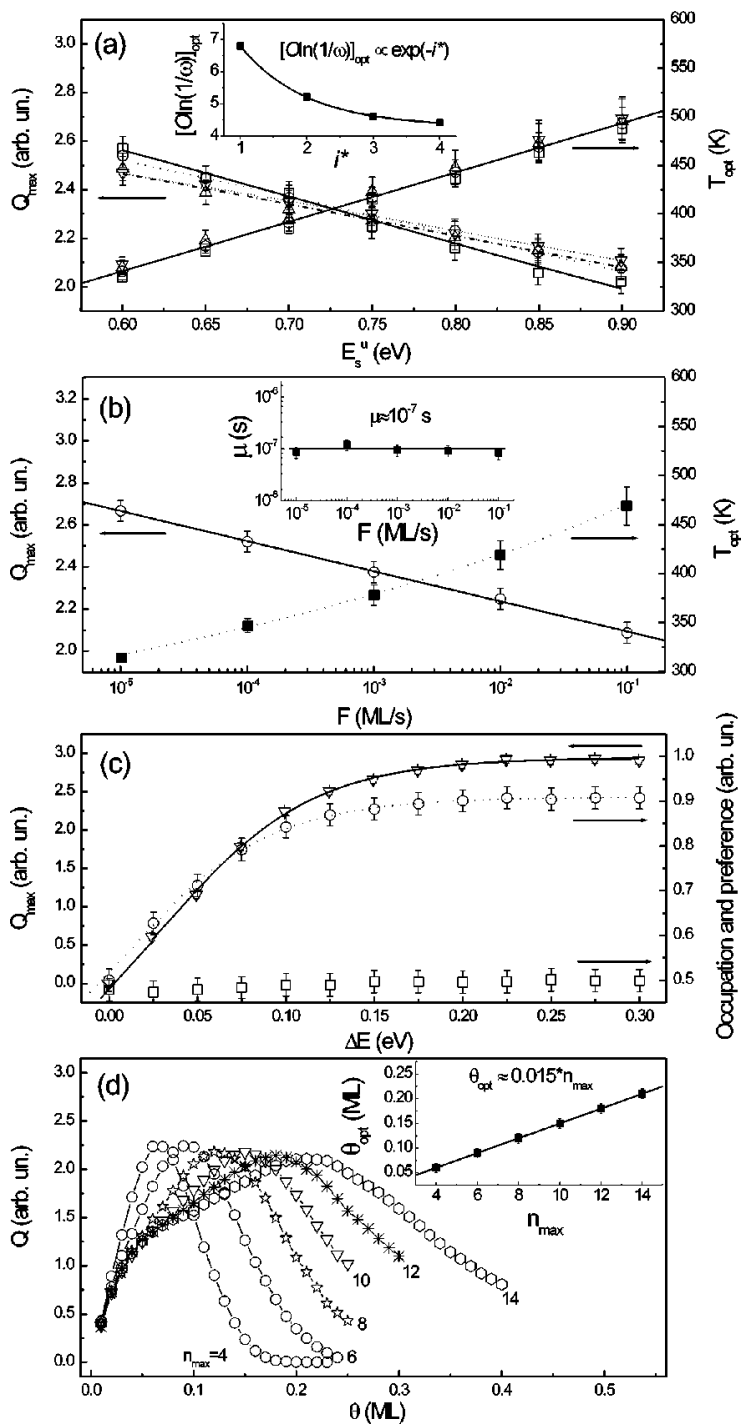

FIG. 2. kMC-simulated dependencies between the optimized deposition/ annealing conditions and the characteristics of metal/ $\mathrm{Si}(111) 7 \times 7$ systems. The parameters are alternatively modified from a prototype set of parameters $\left(E_{s}^{u}=0.75 \mathrm{eV}, \Delta E=0.10 \mathrm{eV}, E_{b}=0.10 \mathrm{eV}, F=0.01 \mathrm{ML} / \mathrm{s}, n_{\max }=6\right.$, and $i^{*}=1$ ) as follows: (a) $E_{s}^{u}$ dependence of $Q_{\max }$ (left axis) and $T_{\text {opt }}$ (right axis) for different $i^{*}$ values $\left(i^{*}=1 \square, 2 \bigcirc, 3 \Delta\right.$, and $\left.4 \nabla\right)$. (b) Flux dependence of $Q_{\max }$ (left axis, $\bigcirc$ ) and $T_{\text {opt }}$ (right axis, $\mathbf{0}$ ). (c) $\Delta E$ dependence of $Q_{\max }$ (left axis, $\nabla$ ), $f^{N}$ (right axis, $\bigcirc$ ) and $O$ (right axis, $\square$ ). (d) $Q(\theta)$ for different $n_{\max }$ values specified in the graph. The symbols correspond to the simulated data whereas the lines represent the fitted analytical dependencies (Table I). The insets in (a) and (b) show the evolution of the fitting parameters $[O \ln (1 / \omega)]_{\mathrm{opt}}$ and $\mu$, respectively, while inset in (d) displays the $\theta_{\mathrm{opt}}\left(n_{\max }\right)$ dependence.

Model: The metal/Si(111) $7 \times 7$ growth has been numerically studied by kinetic Monte Carlo (kMC) simulation ${ }^{10}$ under the following assumptions: ${ }^{11}$ (i) A uniform flux ( $F$ in $\mathrm{ML} / \mathrm{s}$ ) of thermally evaporated particles (with kinetic energies in the meV range) on the $\mathrm{Si}(111) 7 \times 7$ surface. The surface is modeled as a discrete point lattice, where each point corresponds to one half cell such that intra-half scale phenomena are ignored. (ii) An inter-half cell cooperative diffusion of monomers biased by nearest neighboring adsorbates (via nonlocal attractive interactions) that takes place at a jump rate ${ }^{11} \propto \nu_{0} \exp \left[-\left(E_{s}^{f / u}+\left(n_{i}-1-k n_{j}\right) E_{b}\right) / k_{B} T\right]$. Here $\nu_{0}$ $=5 \times 10^{9} \mathrm{~s}^{-1}$ (Refs. 11 and 12) corresponds to the preexponential factor; $E_{s}^{f f u}$ is the hopping energy barrier to escape from faulted/unfaulted half cells whose difference is $\Delta E$ $=E_{s}^{f}-E_{s}^{u} \neq 0 ; n_{i}\left(n_{j}\right)$ is the size of the cluster nucleated on the Downloaded 21 Dec 2006 to 134.94.122.39. Redistribution subject

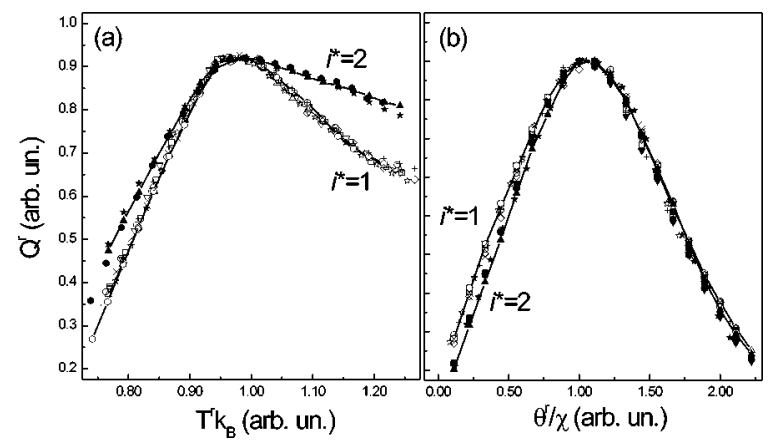

FIG. 3. Overlap of kMC-simulated $Q^{r}$ curves [plotted vs $T^{r} k_{B}$ (a) and $\theta^{r} \chi^{-1}$ (b) for the corresponding $\theta_{\mathrm{opt}}$ and $T_{\mathrm{opt}}$, respectively] for a broad spectrum of parameter values. The parameters are alternatively modified from a prototype set of parameters $\left(E_{s}^{u}=0.75 \mathrm{eV}, \Delta E=0.10 \mathrm{eV}, E_{b}=0.10 \mathrm{eV}, \quad F\right.$ $=0.01 \mathrm{ML} / \mathrm{s}, n_{\max }=6$ and $\left.i^{*}=1\right)$ as follows: $E_{s}^{u}$ series ( $\square / \mathbf{\square}, 0.65 ; \Delta / \mathbf{\Delta}$, 0.75 ; and, $\bigcirc 0.85 \mathrm{eV}), \Delta E$ series $(\diamond, 0.05 ; \triangle / \mathbf{\Delta}, 0.10 ; \boldsymbol{\nabla} / \nabla, 0.15 \mathrm{eV}), F$ series (open and solid hexagons, $0.001 ; \triangle / \mathbf{\Delta}, 0.01$; and,$+ 0.1 \mathrm{ML} / \mathrm{s}$ ), and $n_{\max }$ series $(\times, 4 ; \triangle, 6$; open and closed stars, 8$)$ for $i^{*}=1$ (open and arealess symbols) and $i^{*}=2\left(Q^{r}\right.$-normalized closed symbols).

source (target) half cell; $E_{b}$ is the effective intra-half cell binding energy; and $k$ represents a spatial attenuation factor for the nonlocal substrate-mediated interactions. (iii) The dissociation of clusters with size $\leqslant i *$ (subcritical clusters) such that the aggregation to such clusters becomes reversible. (iv) The existence of a maximum capacity $\left(n_{\max }\right)$ of adsorbates per half cell ${ }^{11,12}$ giving rise to blocking and bouncing back phenomena from half cells saturated by maximum-size clusters. (v) A postdeposition relaxation to take into account the delay between the stages of evaporation and sample characterization (here $4 \mathrm{~h}$ ).

System inspection: Once the model has been depicted, the array formation process is described by the statistical characteristics of the system averaged over 25 runs. These characteristics are: (i) the surface occupation $(O)$, defined as the ratio of the number of occupied half cells to the total number of half cells in the simulated surface and comprising both faulted $(f)$ and unfaulted $(u)$ contributions $(O=f+u)$; and (ii) faulted $\left(f^{N}\right)$ and unfaulted $\left(u^{N}\right)$ occupation preferences described as $f^{N}=f / O$ and $u^{N}=u / O\left(f^{N}+u^{N}=1\right)$. From these definitions, the misalignment and filling defects can be connected with the nucleation on unfaulted half cells and the density of empty faulted half cells, respectively, such that the terms in Eq. (1) are identified as $(1-\alpha)=2\left(f^{N}-1 / 2\right)$ and $(1$ $-\beta)=2 f=2 O f^{N}$. On the other hand, a $g(1 / \omega)=\ln (1 / \omega)$ function has been chosen to describe in a sensitive but moderate way the statistical weight of the uniformity defects in metal $\mathrm{Si}(111) 7 \times 7$. Thus, for the metal cluster selforganization on $\operatorname{Si}(111) 7 \times 7, Q$ can be rewritten as

$$
Q=-4 O f^{N}\left(f^{N}-0.5\right) \ln (\omega) .
$$

The so-defined figure of merit depends implicitly on the system intrinsic characteristics as well as on the deposition/ annealing parameters. As part of a large simplification a total of seven parameters forming a seven-dimensional space have been here considered. This space has been explored in different directions ${ }^{13}$ (with $\theta=0-0.4, \quad T=73-573 \mathrm{~K}, \quad F$ $=10^{-5}-10^{-1} \mathrm{ML} / \mathrm{s}, E_{s}=0.60-0.90 \mathrm{eV}, \Delta E=0-0.30 \mathrm{eV}, i^{*}$ $\left.=1-4, n_{\max }=4-14\right)$ for $Q$ maximum conditions avoiding possible saddle points for the deposition/annealing parameters $(T, F$, and $\theta)$. The results of such explorations are shown in Figs. 1 and 2. Figure 1 shows the $Q(T, \theta)$ surface to AIP license or copyright, see http://apl.aip.org/apl/copyright.jsp 
TABLE I. Found analytical dependencies between the optimized deposition/annealing parameters and the characteristics of the metal/ $\mathrm{Si}(111) 7 \times 7 \mathrm{systems}$. a, b, c and d correspond to dependencies shown in Figs. 2(a)-2(d), respectively.

\begin{tabular}{lccc}
\hline \hline & $\theta_{\mathrm{opt}}$ & $T_{\mathrm{opt}}$ & $Q_{\text {max }}$ \\
\hline$E_{s}^{u}$ & $\partial \theta_{\mathrm{opt}} / \partial E_{s} \approx 0$ & $T_{\mathrm{opt}}=-E_{s}^{u} / k_{B} \ln (\mu F), \mu \approx 10^{-7} \mathrm{~s} 2(\mathrm{a}), 2(\mathrm{~b})$ & $Q_{\max } \approx 4[O \ln (1 / \omega)]_{\mathrm{opt}} f_{\mathrm{opt}}^{N}\left(f_{\mathrm{opt}}^{N}-1 / 2\right)$ \\
$F$ & $\partial \theta_{\mathrm{opt}} / \partial F \approx 0$ & $\partial T_{\mathrm{opt}} / \partial i^{*} \approx 02(\mathrm{a})$ & $f_{\mathrm{opt}}^{N} \approx\left[1+\exp \left(-\Delta E / k_{B} T_{\mathrm{opt}}\right]^{-1} 2(\mathrm{c})\right.$ \\
$i^{*}$ & $\partial T_{\mathrm{opt}} / \partial i^{*} \approx 0$ & $\partial T_{\mathrm{opt}} / \partial \Delta E \approx 0$ & {$[O \ln (1 / \omega)]_{\mathrm{opt}} \propto \exp \left(-i^{*}\right) 2(\mathrm{a})$} \\
$\Delta E$ & $\partial \theta_{\text {opt }} / \partial \Delta E \approx 0$ & $\partial T_{\mathrm{opt}} / \partial n_{\max } \approx 0$ & $\partial Q_{\max } / \partial n_{\max } \approx 02(\mathrm{~d})$ \\
$n_{\max }$ & $\theta_{\mathrm{opt}}=\chi n_{\text {max }}, \chi \approx 0.0152(\mathrm{~d})$ & & \\
\hline \hline
\end{tabular}

for a prototype set of parameters used along the text (see Figs. 2 and 3). The surface features a maximum around the optimal deposition conditions defined (in this case) as $T_{\text {opt }}$ $=420 \pm 10 \mathrm{~K}$ and $\theta_{\text {opt }}=0.09 \pm 0.01 \mathrm{ML}$. The most significant outcomes concerning the kMC-performed optimization process are summarized in Fig. 2 (symbols) while the fitted analytical dependencies (lines) are compiled in Table I. The $x$-independence of the $y$ parameter (with $x$ and $y$ taking generic values) within the explored parameter space is indicated as: $\partial y / \partial x \approx 0$. The optimization relationships of the deposition/annealing conditions with the system intrinsic characteristics to be outlined are the following: (i) The $T_{\text {opt }}\left(E_{s}^{u}, F\right)$ dependence shown separately in Figs. 2(a) and 2(b) describes the necessity of increasing the deposition/ annealing temperature to strengthen the system relaxation to the thermodynamical equilibrium state at relatively short times as the kinetic limitations induced by random incidence and/or the high surface corrugation are enhanced ${ }^{8}$ (i.e., $F$ and $E_{s}^{u}$ increase, respectively). Thus, the existence of a fitting constant $\mu \approx 10^{-7} \mathrm{~s}$ [Fig. 2(b) inset] indicates the found $T_{\text {opt }}\left(E_{s}^{u}, F\right)$ dependence (Table I, first cell at $T_{\text {opt }}$ column) satisfies the equilibrium condition $\nu \exp \left(-E_{s}^{u} / k_{B} T_{\text {opt }}\right) / F$ $=\nu \mu$ pointing to a constant ratio of the diffusion rate to deposition rate. $Q$ decreases as $T_{\text {opt }}$ increases according to the equilibrium relation that predicts a reduction of occupation preference with the temperature [see below the $f^{N}(\Delta E)$ dependence]. (ii) The $i^{*}$ dependence of $Q$ shown in Fig. 2(a) (main and inset) and compiled in Table I (first cell at $Q_{\max }$ column) is related to kinetic limitations imposed by the reversible aggregation into large quasi-immobile clusters, ${ }^{8}$ which retard the system relaxation rate. (iii) The $\Delta E$ dependence of $Q$ [Fig. 2(c) and Table I, first cell at $Q_{\max }$ column] is given through the detailed balance condition for the mobile species [i.e., the monomers: $\rho_{1}^{f} \nu \exp \left(-E_{s}^{f} / k_{B} T\right)$ $=\rho_{1}^{u} \nu \exp \left(-E_{s}^{u} / k_{B} T\right)$, where $\rho_{1}^{f / u}$ corresponds to the monomer density in faulted/unfaulted half cells] which rules the occupation preference ${ }^{8} f^{N} \propto \rho_{1}^{f} /\left(\rho_{1}^{f}+\rho_{1}^{u}\right)=\left[1+\exp \left(-\Delta E / k_{B} T\right)\right]^{-1}$ once the system is relaxed. (iv) Finally, the $Q\left(n_{\max }\right)$ dependence in Fig. 2(d) follows the scaling relationships of $\langle n\rangle$ $\times(\theta)$ and $\omega(\theta)$ with $n_{\max }$ giving rise to a linear increase of $\theta_{\text {opt }}$ as $n_{\text {max }}$ rises [Fig. 2(d) inset and Table I, last cell at $\theta_{\text {opt }}$ column].

It should be stressed that $Q$ scales with the system intrinsic characteristics, for a defined $i^{*}$ value, as

$$
\begin{gathered}
Q^{r}\left(T^{r}, \theta^{r}, i^{*}\right)=\left[f_{\mathrm{opt}}^{n}\left(f_{\mathrm{opt}}^{n}-\frac{1}{2}\right)\right] Q\left[T^{r}=\frac{T}{E_{s}} \ln \left(\frac{1}{\mu F}\right),\right. \\
\left.\theta^{r}=\frac{\theta}{n_{\max }}, \frac{F}{F}, \frac{E_{s}}{E_{s}}, \frac{\Delta E}{\Delta E}, i^{*}, \frac{n_{\max }}{n_{\max }}\right],
\end{gathered}
$$

such that the seven-dimensional space reduces to an "univer- sal" tri-dimensional space, which is common for any metal/ $\mathrm{Si}(111) 7 \times 7$ system. Such a space would be characterized by reduced deposition/annealing parameters $T^{r}$ and $\theta^{r}$ as revealed by the overlap of the open/arealess symbol curves in Fig. 3. $Q$ does not scale with $i^{*}$ (i.e., the form of the $Q^{r}$ curves modifies as $i^{*}$ changes) as shown in Fig. 3 on comparison of the open/arealess symbol curves with the closed symbol ones for $T^{r} \neq 1 / k_{B}$ and $\theta^{r}<\chi$, respectively. The $r e$ duced figure of merit would explain the perfection of the In cluster arrays achieved by $\mathrm{Li}$ et $a .^{2}$ by assuming $E_{s}^{u}$ $=0.75 \pm 0.05 \mathrm{eV}$ and $n_{\max }=8$ (parameters not reported in Ref. 2 ). In any case, systematic experimental studies are required to validate the optimization procedure presented here.

In conclusion, the proposed figure of merit gathers the most significant defects inherent in 1D/2D arrays of selforganized nanoelements and constitutes a worthy tool with scaling properties to optimize the deposition/fabrication conditions of such arrays. Its usefulness has been theoretically demonstrated on self-organized metal/Si(111) $7 \times 7$ systems, in which the main optimization relationships between the deposition/annealing parameters and the system characteristics have been revealed through a simple model.

The author thanks V. Nagarajan and M. Davis for a critical reading of the manuscript.

${ }^{1}$ L. Vitali, M. G. Ramsey, and F. P. Netzer, Phys. Rev. Lett. 83, 316 (1999). ${ }^{2}$ J.-L. Li, J.-F. Jia, X.-J. Liang, X. Liu, J.-Z. Wang, Q.-K. Xue, Z.-Q. Li, J. S. Tse, Z. Zhang, and S. B. Zhang, Phys. Rev. Lett. 88, 066101 (2002).

${ }^{3}$ R. Plass, J. A. Last, N. C. Bartelt, and G. L. Kellogg, Nature (London) 412, 875 (2001).

${ }^{4}$ P. Venezuela, J. Tersoff, J. A. Floro, E. Chanson, D. M. Follstaedt, F. Liu, and M. G. Lagally, Nature (London) 397, 678 (1998).

${ }^{5}$ H. Brune, M. Giovannini, K. Bromann, and K. Kern, Nature (London) 394, 451 (1998).

${ }^{6}$ I. Szafraniak, C. Harnagea, R. Scholz, S. Bhattacharyya, D. Hesse, and M. Alexe, Appl. Phys. Lett. 83, 2211 (2003); E. Vasco, S. Karthäuser, R. Dittmann, J. Q. He, C. L. Jia, K. Szot, and R. Waser, Adv. Mater. (in press).

${ }^{7}$ Considering interstitial sites aligned on an embedded lattice.

${ }^{8}$ E. Vasco, Phys. Rev. B 69, 075412 (2004).

${ }^{9}$ J. Dabrowski and H-J. Mússig, Silicon Surfaces and Formation of Interfaces (World Scientific, Singapore, 2000).

${ }^{10} \mathrm{kMC}$ simulations were performed on $256 \times 256$ half-cell lattice with periodic boundary conditions.

${ }^{11}$ E. Vasco, C. Polop, and E. Rodríguez-Cañas, Phys. Rev. B 67, 235412 (2003).

${ }^{12}$ J. Mysliveček, P. Sobotík, I. Ošt'ádal, T. Jarolímek, and P. Šmilauer, Phys. Rev. B 63, 045403 (2001)

${ }^{13}$ The maximum $Q$ conditions within the seven-dimensional space are determined by numerical recipes making use of the gradient and the function value to perform a 1D line maximization procedure. 